\title{
Lifestyle and its Potential to Preserve Cognitive Function and Reduce the Risk of Dementia
}

\author{
An Expert Interview with Maria C Carrillo
}

Chief Science Officer, Medical \& Scientific Relations, Alzheimer's Association, Chicago, IL, US

DOI: https://doi.org/10.17925/USN.2018.14.1.11

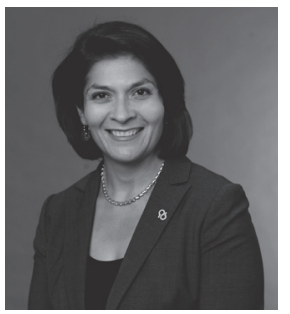

\section{Maria C Carrillo}

Maria Carrillo, PhD, is Chief Science Officer, Medical and Scientific Relations at the Alzheimer's Association. Dr. Carrillo has a wide range of responsibilities, including oversight of the Association's grant-making process and communication of scientific findings within and outside of the organization. Dr. Carrillo directly manages several Alzheimer's Association initiatives including the Research Roundtable, the World Wide Alzheimer's Disease Neuroimaging Initiative, and the Global Alzheimer's Association Interactive Network. She is co-author of the National Institute on Aging-Alzheimer's Association revised criteria for the diagnosis of Alzheimer's, and the Appropriate Use Criteria for Amyloid Imaging. Dr. Carrillo was the Alzheimer's Expert Scientific Consultant for the movie Still Alice (2014). In 2016, Dr. Carrillo joined the Governing Board of the Global Brain Health Institute, which supports a new generation of leaders to translate research evidence into effective policy and practice. In 2016, Dr. Carrillo also became a member of the Research Committee for the American Heart Association.

\section{Keywords}

Alzheimer's disease, brain health, cognitive function, dementia, lifestyle, prevention, risk factors

Disclosure: Maria C Carrillo has nothing to declare in relation to this article.

Review Process: This is an expert interview and as such, has not undergone the journal's standard peer review process.

Authorship: The named author meets the Internationa Committee of Medical Journal Editors (ICMJE) criteria for authorship of this manuscript, takes responsibility for the integrity of the work as a whole, and has given final approval for the version to be published.

open Access: This article is published under the Creative Commons Attribution Noncommercial License, which permits any noncommercial use, distribution, adaptation, and reproduction provided the original author and source are given appropriate credit. (c) The Author 2018.

Received: January 17, 2018

Published Online: February 16, 2018

Citation: US Neurology. 2018;14(1):11-13

Corresponding Author: Maria C Carrillo, Alzheimer's Association, 225 N. Michigan Ave., Fl. 17, Chicago, IL 60601-7633, US. E: mcarrillo@alz.org Twitter: @DrMariaALZ

Support: No funding was received in the publication of this article.
$\mathrm{T}$ he annual Alzheimer's Association International Conference (AAIC) took place in London, UK in July 2017. In an expert interview, the Alzheimer's Association's very own Maria Carrillo, Chief Science Officer, Medical and Scientific Relations, shares her perspectives on one of the key themes of the meeting - lifestyle and its potential to reduce the risk of developing dementia.

\section{Q. What proportion of dementia cases could be preventable through lifestyle modification?}

In a report presented at AAIC 2017, The Lancet International Commission on Dementia Prevention, Intervention and Care reported that more than one third (roughly $35 \%$ ) of global dementia cases may be preventable through addressing lifestyle factors that impact an individual's risk. ' These potentially modifiable risk factors have been identified across the life-span, not just in old age.

The Lancet Commission's report was simultaneously published in The Lancet and presented at AAIC 2017.1,2 The Commission's report recommended targeted public health strategies that the researchers expect will significantly lower the global burden of Alzheimer's and other dementias. For example:

- The authors strongly recommend vigorously treating hypertension in middle aged and older people without dementia to reduce dementia incidence.

- Other recommended interventions include more childhood education, getting regular exercise, maintaining social engagement, stopping smoking, and management of hearing loss, depression, diabetes, and obesity.

The Alzheimer's Association has launched the US Study to Protect Brain Health Through Lifestyle Intervention to Reduce Risk (US POINTER), a two-year clinical trial to test whether lifestyle interventions focused on combining healthy nutrition, physical activity, social and intellectual challenge, and increased medical monitoring of vascular and metabolic conditions can protect cognitive function in older adults who are at increased risk for cognitive decline. ${ }^{3}$ US POINTER is the first study to examine this combined multi-dimensional intervention in a large-scale US-based population. In total, 2,500 participants age 60-79 at high risk for dementia will be enrolled. 


\section{Q. What are the most important dietary factors associated with preservation of cognitive function?}

Eating a healthful diet benefits both your body and your brain. ${ }^{4}$ In general, this is a diet that is lower in saturated fats and higher in fruits and vegetables, whole grains, nuts and fish. While research on the relationship between diet and cognitive functioning is somewhat limited, it does point to the benefits of two diets in particular: the Dietary Approaches to Stop Hypertension (DASH) diet and the Mediterranean diet. These diets can help reduce heart disease and may also reduce risk of cognitive decline and dementia.

Research has suggested that combining good nutrition with mental, social and physical activities may have a greater benefit in maintaining or improving brain health than any single activity. At AAIC 2014, a two-year clinical trial of older adults at risk for cognitive impairment showed that a combination of physical activity, nutritional guidance, cognitive training, social activities and management of heart health risk factors slowed cognitive decline. ${ }^{5}$

\section{Q. What were the findings of the recent study examining the association between verbal fluency and cognitive decline?}

In an analysis of participants in the Wisconsin Registry for Alzheimer's Prevention (WRAP), researchers investigated whether people with very early memory decline also showed changes in their everyday speech. The researchers found that subtle changes in everyday speech, such as the increased use of short sentences, more pronouns, and pauses like "um" and "ah," correlated with early mild cognitive impairment (eMCl), which can be a precursor to Alzheimer's disease.

In the study reported at AAIC 2017, the scientists analyzed two speech samples, taken two years apart, from 264 participants in WRAP. Of these participants, 64 were identified as having eMCl based on cognitive testing over 8-10 years. The speech samples, averaging one minute long, were collected by asking the participants to describe a simple picture. ${ }^{6}$

Study participants with eMCl declined faster on two measures of speech: content and fluency. The content of their speech was less specific, with a higher proportion of pronouns to nouns (e.g., "she," "it," "them"). Their fluency was more disrupted (more hesitations, word repetitions, and filled pauses ("um," "uh"). Also, those with eMCl used less complex syntax and shorter sentences, and took more time to express the same amount of content as the cognitively healthy group. ${ }^{6}$

Confirmation and replication are needed, but the scientists suggest that speech analysis may be a valuable cognitive marker to add to clinical assessments of cognitive function in the future. ${ }^{6}$

\section{Q. Could you tell us a little about racial disparities in the incidence and risk of Alzheimer's disease?}

According to the Alzheimer's Association 2017 Alzheimer's Disease Facts and Figures report, a review of many studies by an expert panel concluded that older African-Americans are about twice as likely to have Alzheimer's or other dementias as older whites, and Hispanics are about one and one-half times as likely to have Alzheimer's or other dementias as older whites.
Currently, there is not enough evidence from population-based cohort studies in which everyone is tested for dementia to estimate the national prevalence of Alzheimer's and other dementias in other racial and ethnic groups. However, a study examining electronic medical records for members of a large health plan in California indicated that dementia incidence - determined by the presence of a dementia diagnosis in one's medical record-was highest in African-Americans, intermediate for Latinos (the term used in the study for those who self-reported as Latino or Hispanic) and whites, and lowest for Asian-Americans. ${ }^{8}$

A series of studies reported at AAIC 2017 in London confirmed racial inequities in numbers of people with Alzheimer's disease and other dementias - even after age 90-and also pointed to growing evidence that early life stress and neighborhood conditions contribute to dementia risk in late life..$^{8-11}$

The studies reported at AAIC 2017 were based on US data, but they add weight to the global body of evidence around disadvantage and dementia risk, which is an issue that governments around the world grapple with, and one that requires coordinated action. For a racially diverse nation like the US, these findings support the need for targeted interventions, whether preventive or service-driven, to help address the gaps we know exist-and for more research.

\section{Q. How can the treatment of sleep disorders impact on the risk of Alzheimer's disease?}

Several new research analyses reported at AAIC 2017 in London found significant associations between sleep-disordered breathing (SDB) and the accumulation of biomarkers for Alzheimer's disease. ${ }^{12-14}$ These findings highlight the idea that SDB is a modifiable factor that may help lower the risk of dementia and possibly slow the progression of dementia where it already exists.

Research reported at AAIC 2017 found:

- SDB accelerated the accumulation of brain $\beta$-amyloid both in cognitively normal individuals and individuals with mild cognitive impairment (data from the Alzheimer's Disease Neuroimaging Initiative $[\mathrm{ADNI}]) .^{14}$

- Obstructive sleep apnea (OSA) was associated with increased brain $\beta$-amyloid deposition, decreased cerebrospinal fluid (CSF) levels of $\beta$-amyloid and increased tau protein levels. ${ }^{13,14}$

The US Centers for Disease Control and Prevention says more than one third of American adults do not get enough sleep on a regular basis. Clearly this is not good for brain health or overall health. ${ }^{15}$

SDB is treatable in many cases. Through early diagnosis and effective treatment of these sleep disorders, there is the potential to improve cognition and possibly reduce dementia risk. However, we need more research before we can say with certainty that sleep interventions have an impact on cognition. In addition, we need to know more about the connections between these medical conditions.

\section{Q. What other modifiable risk factors for cognitive decline do you consider important?}

In 2015, the Alzheimer's Association published, "Summary of the evidence on modifiable risk factors for cognitive decline and dementia: 
a population-based perspective," in Alzheimer's \& Dementia: The Journal of the Alzheimer's Association. ${ }^{16}$ This was in response to a request from the World Dementia Council (WDC) for the Association to evaluate and report on the state of the evidence on modifiable risk factors for cognitive decline and dementia to support the WDC in any future recommendations.

In that article, the Alzheimer's Association stated its belief that there is sufficient evidence to support the link between several modifiable risk factors and a reduced risk for cognitive decline, and sufficient evidence to suggest that some modifiable risk factors may be associated with reduced risk of dementia.
Specifically, the Association believes there is sufficiently strong evidence, from a population-based perspective, to conclude that regular physical activity and management of cardiovascular risk factors (diabetes, obesity, smoking, and hypertension) reduce the risk of cognitive decline and may reduce the risk of dementia. The Association also believes there is sufficiently strong evidence to conclude that a healthy diet and lifelong learning/cognitive training may also reduce the risk of cognitive decline.

The US POINTER study, mentioned earlier, will take this a significant step further by testing, in a large-scale, two-year randomized controlled clinical trial, the impact of a multi-component lifestyle intervention on protecting cognitive function in older adults who are at increased risk for cognitive decline.
1. Ritchie K, Livingston G, Howard RJ, Schneider LS. Focused Topic Session: The Lancet International Commission on Dementia Prevention and Care. Presented at: Alzheimer's Association International Conference, London, UK, 20 July 2017. Abstracts available at: Alzheimers Dement. 2017;13 Suppl. P1448-50.

2. Livingston $\mathrm{G}$, Sommerlad $\mathrm{A}$, Orgeta $\mathrm{V}$, et al. Dementia prevention, intervention, and care. Lancet. 2017;390:2673-734.

3. Alzheimer's Association. US Study to Protect Brain Health Through Lifestyle Intervention to Reduce Risk (US POINTER). Information available at: https://alz.org/us-pointer/ (accessed January 24, 2018)

4. Alzheimer's Association. Brain heath: adopt a healthy diet. Available at: www.alz.org/brain-health/adopt healthy diet asp (accessed January 24, 2018)

5. Solomon $\mathrm{A}$, Levälahti $\mathrm{E}$, Soininen $\mathrm{H}$, et al. A multidomain, two-year, randomized controlled trial to prevent cognitive impairment: the FINGER study. Alzheimers Dement. 2014;10 Suppl. P137-8.

6. Mueller KD, Koscik RL, Turkstra LS, et al. Signs of early cognitive decline within connected speech: evidence from the Wisconsin
Registry for Alzheimer's Prevention (WRAP). Alzheimers Dement. 2017;13 Suppl. P812-14.

7. Alzheimer's Association. 2017 Alzheimer's disease facts and figures. Alzheimers Dement. 2017;13:325-73.

8. Whitmer RA, Gilsanz P, Kawas $\mathrm{CH}$, et al. Racial/ethnic differences in rates of dementia incidence among the oldest-old. Alzheimers Dement. 2017:13 Suppl. P195.

9. Gilsanz P, Mayeda ER, Glymour M, et al. Birth in a high infant mortality state: race and risk of dementia. Alzheimers Dement. 2017;13 Suppl. P210-11.

10. Zuelsdorff $M$, Gleason CE, Kind AJ, et al. Lifetime stressful experiences, racial disparities, and cognitive performance: findings from the Wisconsin Registry for Alzheimer's Prevention (WRAP) study. Alzheimers Dement. 2017:13 Suppl. P212.

11. Kind AJ, Bendlin BB, Kim AJ, et al. Neighborhood socioeconomic contextual disadvantage, baseline cognition and Alzheimer's disease biomarkers in the Wisconsin Registry for Alzheimer's Prevention (WRAP) study. Alzheimers Dement. 2017;13 Suppl. P195-6.
12. Shim A, Hogan M, Halldin K, et al. Sleep disordered breathing APOE 4 and $\beta$-amyloid deposition in cognitive normal elderly. Alzheimers Dement. 2017;13 Suppl. P16.

13. Hogan M, Shim A, Halldin K, et al. Obstructive sleep apnea is associated with longitudinal increases in amyloid burden in elderly mild cognitive impairment individuals. Alzheimers Dement. 2017;13 Suppl. P15-6.

14. Bubu OM, Sharma RA, Umasabor-Bubu OQ, et al. Effect of obstructive sleep apnea (OSA) on rate of change of $A D$ biomarkers in cognitively normal, $\mathrm{MCl}$ and $\mathrm{AD}$ elderly: findings from the Alzheimer's Disease Neurolmaging Initiative (ADNI) cohort. Alzheimers Dement. 2017;13 Suppl. P1008-9.

15. Centers for Disease Control and Prevention (CDC). 1 in 3 adults don't get enough sleep. Available at: www.cdc.gov/media/ releases/2016/p0215-enough-sleep.html (accessed January 24 2018).

16. Baumgart M, Snyder HM, Carrillo MC, et al. Summary of the evidence on modifiable risk factors for cognitive decline and dementia: a population-based perspective. Alzheimers Dement. 2015;11:718-26 